\title{
Dist-Coop: Distributed Cooperative Transmission in UWSNs using Optimization Congestion Control and Opportunistic Routing
}

\author{
Malik Taimur Ali \\ Department of Computer Science \\ Iqra National University \\ Peshawar, Pakistan \\ Saqib Shahid Rahim \\ Department of Computer Science \\ Abasyn University \\ Peshawar, Pakistan
}

\author{
Mian Ahmed Jan \\ Department of Computer Science \\ Abdul Wali Khan University \\ Mardan, Pakistan \\ Atif Ishtiaq \\ Department of Computer Science \\ Iqra National University \\ Peshawar, Pakistan
}

\author{
Sheeraz Ahmed \\ Department of Computer Science \\ Iqra National University \\ Peshawar, Pakistan \\ Mukhtar Ahmad \\ Department of Electronics \\ Islamia College Peshawar \\ Peshawar, Pakistan
}

\author{
Mukhtaj Khan \\ Department of Computer Science \\ Abdul Wali Khan University \\ Mardan, Pakistan
}

\author{
M. Ayub Khan \\ Department of Computer Science \\ Iqra National University \\ Peshawar, Pakistan
}

\begin{abstract}
One of the real issues in UWSN is congestion control. The need is to plan an optimized congestion control scheme which enhances the network life time and in addition limits the usage of energy in data transmission from source to destination. In this paper, we propose a routing protocol called Dist-Coop in UWSN. Dist-Coop is a distributed cooperation based routing scheme which uses mechanism for optimized congestion control in noisy links of underwater environment. It is compact, energy proficient and high throughput opportunistic routing scheme for UWSN. In this proposed protocol architecture, we present congestion control with cooperative transmission of data packets utilizing relay sensors. The final objective is to enhance the network life time and forward information utilizing cooperation procedure, limiting energy consumption amid transmission of information. At destination node, combining strategy utilized is based on Signal-to-Noise Ratio (SNRC). Simulation results of Dist-Coop scheme indicate better outcomes in terms of energy consumption, throughput and network lifetime in contrast with Co-UWSN and EH-UWSN routing protocols. Dist-Coop has expended substantially less energy and better throughput when contrasted with these protocols.
\end{abstract}

Keywords-Opportunistic routing; cooperation; congestion control; signal-to-noise ratio

\section{INTRODUCTION}

Wireless networks are such type of networks which use Infrared or Radio Frequencies signals to share data or information between the devices connected with each other. There are number of wireless devices available now a days; for example, laptops, mobile sets, small size PCs, wireless sensors and satellite receivers among others. The new fourth era of cellular communication has greatly increased the data transmission speed, which provides the variety of high speed mobile data rates. At the same time, different new standards like Bluetooth, Infrared, 802.11 for short range radio helps to produce a variety of new application for enterprises and home networking, which enables wireless multimedia and data communication in offices and homes. Examples of wireless networks are cellular networks, Terrestrial Networks, Satellite Communication Networks, Ad hoc Networks and Wireless Sensor Networks [1].

\section{A. Types of Wireless Sensor Networks}

Sensor nodes are most commonly deployed in Remote areas and underwater. In these areas, the sensor network faces different challenges according to the environment. Mainly there are five types of WSNs:

1) Terrestrial Wireless Sensor Networks

2) Underground Wireless Sensor Networks

3) Underwater Wireless Sensor Networks

4) Multi-media Wireless Sensor Networks

5) Mobile Wireless Sensor Networks

1) Terrestrial Wireless Sensor Networks: These types of networks mainly consists of hundreds to thousands small inexpensive sensor nodes, which are deployed in a specific region, either in ad hoc (unstructured) manner or in pre-defined (structured) manner. In ad hoc manner, the sensor nodes are deployed in a specific region randomly. In terrestrial WSNs, the sensor nodes must be able to communicate successfully 
with the base station, while limited battery power is available [2].

2) Underground Wireless Sensor Network: In Underground WSNs, the sensor nodes are placed underground in a specific targeted area. Basically it is used to observe the underground situation and communicate this information to the surface area. These sensor nodes transmit the information to the sink nodes, which are further transmitted to the base station. The underground WSNs are more costly as compared to terrestrial WSNs. Due to signal losses and attenuation, the wireless communication becomes a challenge in these different environments. To increase the life time of the network, careful planning and cost consideration is required in underground WSNs [3].

3) Underwater Wireless Sensor Networks: In these types of networks, numbers of nodes are deployed into the water. Underwater WSNs are more costly and very difficult to deploy it in underwater. Sensor nodes in underwater WSNs communicate with each other and with the sink nodes using acoustic signals. Acoustic communication is again big challenge in underwater due to limited bandwidth, large propagation delay, signal fading problems and sensor node failure. These sensor nodes have a limited battery power and very difficult to recharge or replace a battery in harsh environment of water. For efficient use of energy, underwater WSNs are required to develop efficient underwater communication and networking techniques as shown in Fig. 1 [4].

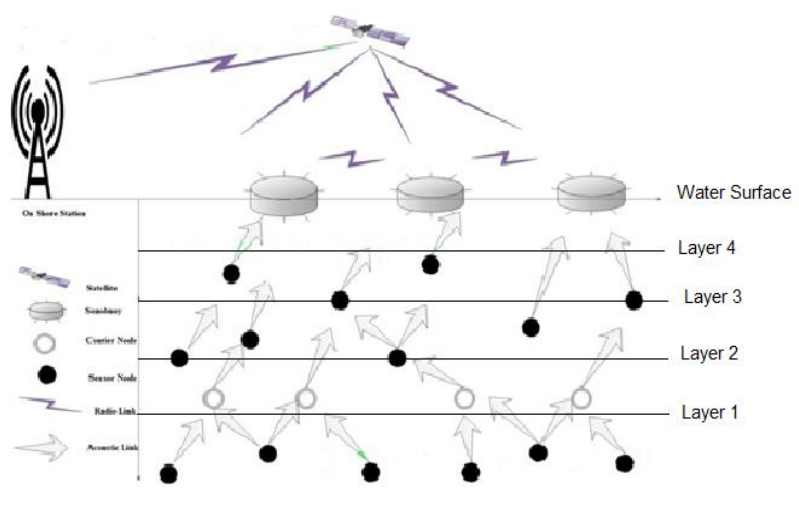

Fig. 1. Underwater wireless sensor network architecture with layers.

4) Multi-media Wireless Sensor Networks: To monitor events or tracking any object, wireless sensor networks are a suitable platform. It consists of low price nodes while having large in number to cover a specific region. These nodes have different properties and connected with each other via different sources. Nodes are arranged in the atmosphere in a pre-defined manner for a specific task [5].

In multi-media applications such as video streaming requires high bandwidth to transmit the whole contents. Therefore, high energy is consumed for high data rates. Multimedia WSNs needs to develop such transmission techniques which have high bandwidth and low energy consumption. Due to variable delay and channel capacity, Quality of Service (QoS) is difficult to preserve in multi-media WSNs. It is necessary to obtain certain level of QoS for reliable delivery of the contents [5].

5) Mobile Wireless Sensor Networks: In such networks, nodes can freely move within the environment on their own capability. These nodes performing different operation like sensing and communicating as a fixed nodes. These nodes have the ability to divert their location and manage themselves accordingly. In a network, mobile node communicates with another mobile node within a range of each other and transfers gathered information [5].

\section{B. Major Challenges in UWSNs}

Quantities of difficulties are looked in brutal submerged conditions. These issues are looked in two angles; Technical and Research challenges, for successful utilization of submerged sensor sensors. A portion of the significant issues are given below [1]:

- Hard to energize batteries and have very limited battery power.

- Accessible bandwidth is limited.

- Channel is influenced by multipath fading.

- Higher size of end to end defer when contrasted with earthly WSN.

- Mistake rates of bits are high.

- Solar energy cannot be used in submerged.

- Corrosion and Fouling may cause to come up short submerged sensors.

- Localizations.

- Data extraction.

One of the significant issues seen in UWSN is battery usage constraint, it's energizing and substitution. Because of unforgiving submerged circumstance, it is exceptionally troublesome and costly to revive or supplant sensor sensors batteries. Based on specified difficulties, the administration and planning of UWSNs routing techniques design is a major test for scientists and researchers [6].

\section{LITERATURE REVIEW}

According to authors in [2], it is exceptionally hard to give effective routing administrations in UWSNs. The radio signals does not work appropriately in underwater condition that's why acoustic signs are utilized. Acoustic signals have lesser transfer speeds and longer spread deferral when contrasted with radio signs. Besides, because of water ebb and flow, Network topology in UWSN in nature is dynamic and sensors move latently. Here the proposed protocol is known as Depth Based Routing convention (DBR) to tackle the issue of restriction up to some degree. DBR does not require data of sensor sensors of full-dimensional area. It requires just neighborhood profundity data that can be effectively 
accomplished by putting a low value profundity sensor with each submerged node.

Researchers in [7], use that a standout amongst the most vital issue in UWSN is energy impediment. Because of unforgiving submerged condition, the charging and substitution of sensor sensors batteries is exceptionally troublesome and costly. The arrangement proposed is to outline an energy effective directing convention named Energy Efficient Depth Based Routing (EEDBR) to take care of the said issue up to some degree in UWSNs. The proposed convention ascertains the profundity of the sensor sensors alongside its lingering energy, to build the life time of the system. Researchers in [8], assess diverse routing conventions like VBF, DBR, H2-DAB, and QELAR and so on based on limitation systems, minimization of energy and ascertain the holding time in UWSNs. Each directing convention has diverse objectives like decrease in energy utilization, accomplishment of strength and so on. This paper for the most part centers to feature the difficulties looked in the plan of directing convention for UWSNs.

Specialist in [9], use that in thick submerged condition, the significant issues are the variety in arrange topology, high blunder rate, and more noteworthy energy utilization for the transmission of information. Expansion with these quantities of issues, there are some real focal points of UWSNs, for example, submerged administration, oil investigations and a few calamities administration issues. Versatile portability of Courier sensors in Threshold-Optimized Depth-based (AMCTD) directing plan has planned to accomplish more noteworthy system lifetime based on sensors profundity, diminishing the utilization of energy particularly amid the steadiness time frame. The versatile development of messenger node maintains the system throughput in the extra state of system. In [10], proposed routing convention in light of sending capacity (FF) for UWSNs named enhanced Adaptive Mobility of Courier sensors in Threshold-Optimized profundity based directing (iAMCTD). Contrasting and the current profundity based routing conventions; developed convention misuses the thickness of the system for time basic data. To beat way misfortune, proliferation dormancy and flooding holding time is ascertained and utilize directing measurements. Which contained confinement free signals to-clamor proportion (LSNR), signals quality list (SQI), Energy cost work (ECF) and Depth Dependent Function (DDF). Reckoning of FF procedure increments the life time of a system and decreases the transmission adversity.

Researchers in [11], used that to diminish the issue of more prominent postponement, the deferral touchy routing convention is required. The proposed plans known as DelaySensitive Depth-Based Routing (DSEEDBR) and affectability based levels having limit esteems to enable the routing in view of its profundity to diminish the three plans are consolidated to configuration defer effective Delay-Sensitive Holding time (DSHT) and Priority Factors (PF). In request to figure the transmission misfortune and got signals speed, ideal weight work (WF) are presented in these plans. Besides, to tackle the issue of postponement, it needs better forward to limit the transmission delay in such locale where sensors are put in low profundity. In DSDBR, WF and Fi are utilized for better forwarder choice and to evaluate DSHT, they presented dth. The significant reason for high engendering delays is low profundity Transmission. Researchers in [12] proposed Dual Sink Efficient and Balanced Energy utilization Technique (DSEBET) for Underwater Acoustic Sensor Networks (UASNs). In UASNs organize the system lifetime crumples because of restricted energy asset. The real issue is to adjust energy in low system lifetime. In this way, to expand the system lifetime, the utilization of energy must be adjusted. In DSEBET, interfaces between the sensors are made based on their little separation "Nj" hand-off node is chosen for information transmission. In transmission of information, each node has parallel Energy Level Numbers (ELNs).

Researchers in [13], proposed impedance mindful and solid directing convention for UWSNs. Based on built up way from end-to-end, the following forwarder has been chosen for transmission of information parcels. Based on this approach, the void gaps can be disposed of utilizing the proposed routing convention. For dependable correspondence, interference with in the divert is additionally considered in directing metric amid the choice of sending node. The proposed directing convention chooses neighbor node as a next forwarder from source to goal, so crash likelihood can be decreased at arrange layer. Later, the momentous progression in the field of UWSNs has been accomplished in [14]. Number of routing conventions is configured to fathom the issues looked in UWSNs. In said plot, the goal is chiefly center to comprehend the working standards of those routing plans. Three investigation strategies are proposed for this reason; Clustering based, limitation based and collaboration based routing. Numerous routing conventions center around energy productivity, security of system and time proficiency diminish the postponement in time and increment the life time of the system.

Researchers in [15], suggested that in agreeable WSN condition, energy proficient plan is utilized as a part of quantities of sensors and Data Gathering Node (DGN). Based on DGN, diverse setup is utilized like the Number of Input to Number of yield and Number of Input to Single Output. In proposed convention, helpful energy plot for MIMO (CMIMO) is utilized, where mistake is rectified by utilizing low thickness equality check (LDPC). The length of LDPC relies upon the measure of the message and mistake checking bits; where the rate of LDPC code changed with the extent of message and equality bits. Analyst in [16], used that in energy constrained systems, Cooperation by utilizing single transfer is more basic and successful correspondence. For the most part center to research and select a hand-off having least energy with control transmission. For agreeable transmission, Relays ascertain their base required power with a specific end goal to oversee collaboration. Just the best one hand-off is chosen among all to diminish the power use. The decision of transfer depends on appropriated path with little overhead.

Authors in [17] offered Energy Harvesting (EH-WSN) technique having the upside of productive collaboration and transfer choice in view of little power use. The convention is assessed in two stages, in initial step, helpful correspondence is assessed and afterward the energy of every node is figured. This plan characterizes unassuming and valuable system for EH-WSN with participation and straightforwardly connected in 
genuine cases. To accomplish energy productive collaboration based correspondence, it can likewise coordinate with different conventions. In [18], proposed to get exceptional capacity by utilizing new form of codes to redesign programming, called shrewd Internet of things (IOT). Because of cruel channel qualities, information gathering is still issues in WSN because of long deferral, energy utilization and retransmission. Information is transmitted agreeably by refreshing programming to accomplish dependable correspondence. Dependability enhanced agreeable correspondence RICC scheme is proposed to enhance the unwavering quality of the system in multi-hop helpful correspondence without diminishing system lifetime. In WSNs, sensors are put at various positions, so every node handle diverse bundles, so uneven energy utilization happened in the system. To proficiently utilize the lingering energy, in RICC, energy utilization is embraced by the prerequisites. Low power utilization is embraced to keep up long system lifetime.

Analysts in [19], argued that the information transmitted by WSNs are utilized for various purposes in various parts like security, arrange topology and correspondence conventions are happens in the sending of WSNs. Distinctive conventions are utilized by the prerequisites of the application, for example, verification, remove, number of parcels transmitted amid particular timeframe. In [20], Author proposed a Wireless Network (WPCN) and the proposed plot comprises of one source to goal combining and having various forward (DF) and translate transfers. This plan proposed energy limit based multi-hand-off choice (ETMRS) for WPCN. The transfers are sent arbitrarily and are known by just its profundity data for switching between Energy and Information Forwarding gathering modes. The limited battery asset of every sensor node, the charging/releasing of node happens that required ETMRS plot over blended Nakagami-m and Rayleigh blurring channels.

In [21], authors proposed a technique expanded the proficiency and unwavering quality of the system by utilizing the sink portability and helpful routing. Numerous conventions are created to perform participation with a specific end goal to enhance connect productivity by examining physical and MAC layer perspectives. The proposed protocols basically center to investigate arrange layer with sink versatility. The goal and hand-off node is chosen based on remaining energy and its profundity data. Sink versatility by social occasion information from various sensors specifically increment the effectiveness of the system. The proposed plot works in various stages; information procurement stage, arrange usage stage, limit based information detecting and directing stage. Every node ascertains its alive neighbor sensors to refresh profundity edge level in organize instatement stage. Researchers in [22], use that to take care of various issues happened in UWSNs, Improved routing arrangement is required for productive information sending. This paper proposed enhanced agreeable plan to build the life time and unwavering quality of the UWSNs. The proposed protocol receives participation on arrange layer inside existing non-agreeable routing plan, Depth Based Routing (DBR), to enhance the dependability and throughput. The determination of hand-off node depends on its profundity and information is sent from source to goal helpfully by utilizing transfer sensors.

In [23], researchers broke down that the cruel submerged condition because of blurring and unavoidable clamors makes it hard to perform mistake free transmission of information. The choice of transfer depends on node profundity and its leftover energy. Analyst in [24], use that to enhance the correspondence quality, agreeable correspondence is received in UWSNs by utilizing transfer sensor sensors. To enhance the execution of the system, proposed a helpful plan known as Analytical approach towards Reliability with Cooperation for UWSNs (ARCUN). The proposed convention has high throughput and energy proficient routing plan for UWSNs. Transfer chose from a gathering of hand-off sensor sensors, ascertain the separation and SNR proportion of the submerged channel. The proposed protocol uses collaboration and SNR for delay-delicate application to expand the parcel conveyance proportion and soundness time of the system. In transmission of information without collaboration implies information is transmitted by utilizing direct connection from source to goal.

In [25], authors use that the two principle challenges are; radio waves can't function admirably in submerged condition and the second is that the acoustic correspondence is moderate. The proposed plot centers around area base collaboration. The region is separated in various clusters and after that collaboration between sensors take place. The proposed protocol named Energy Efficient Adaptive Cooperation Routing convention for UWSNs (EACE) accomplished longer system lifetime and less utilization of energy with participation between sensors. Specialist in [26] use that dependability is one of the imperative factors to enhance the general execution of UWSNs. Uproarious condition and poor channel quality lessening the dependability and influence the system execution by influencing the trustworthiness of information. Helpful directing in UWSNs enhances the unwavering quality and honesty of the information. The proposed protocol called Improved Adaptive Cooperative Routing in UWSNs (IACR), comprises of two transfer node and one ace node is chosen among the accessible sensors from source to sink node for transmission of information.

In [27], researchers recommended that Cooperative condition increment the life time of the system in testing condition. Neighbor sensors are utilized for transmission of information helpfully as transfer sensors. The planned protocol named Cooperative UWSN (Co-UWSN), which builds the system life time by expanding unwavering quality, energy effectiveness and expanding throughput in UWSNs. To beat blurring, agreeable assorted variety is presented. Choice of hand-off node in light of channel quality and separation among neighbor node for quality and separation among neighbor node for fruitful information bundle transmission. Loss of information parcel is diminished by happening variety inside and out limit level. The system soundness period and load adjusting is accomplished by utilizing ideal weight calculation and helpful condition. 


\section{Motivation}

Co-UWSN and SPARCO are proficient plans yet it might have a few issues in adjusting of load in sensors having low profundity. Vast energy is devoured by these low profundity sensors when transmitting information, which create scope gaps in a system. These gaps are essentially created because of load administration among alive sensors. In the event that the profundity expands, the utilization of energy amid transmission is additionally expanded.

All sensor nodes have ability to transfer theirs data as well as data that came from other sensor nodes by the route that ending at sink. Nodes utilize also relay sensor nodes when transferring the data to BS. Before collecting data packets, a routine process performed to decide an optimal node amongst neighbors of each sensor node to minimize cost function. Sensed and incoming data packets traffic is transferred to this sensor node. The establishing of these paths guarantees that collected data are forwarded to BS. Issues of the multi hop routing are attempted here by two stage technique. According to 1st stage, optimum cooperation based transmission approach is imitative for every sensor node for sending data to their neighbor sensor nodes in case of selected. Each sensor node recognizes link cost for its neighbors. In 2nd stage, a routing algorithm is used called Bellman-Ford distributed algorithm for obtaining minimum cost route from any sensor node to BS. Cost values of link attained in 1st stage that is utilized in 2nd stage. Data is labeled by creating sensor nodes and organized as bit data packets.

According to broadcasting stage, source sensor node broadcast data packets with index of destination sensor node. The subset of neighboring relays get signals and in 2nd stage use the cooperation based approach for transmission such as decode and forward strategy to send data packets to destination. It is also possible that source take part too in 2nd stage in case if these outcomes in lesser total consumption of power. Power of transmission in cooperation based transmissions also requires determining. Therefore with fix destination, the source requires to determine broadcast power that is represented by $\mathrm{Pb}$. In addition, SNR at destination is mandatory to be high by set verge that is stated formerly. Generally relays are also used by other sensor nodes in network. Therefore for cooperation based transmission, destination requires to recognize to relays. Hence index of destination is also required to transfer to relays. The main purpose of any cooperation based transmission is delivering data packets from a source to a destination when satisfying SNR, with least likely total power of transmission.

It is also important to note that resolution of this issue may be direct which non-cooperative transmission from source to destination is. It is also be noted that by selecting a greater values of $\mathrm{Pb}$, more sensor nodes are involved in cooperation based transmission that is leads to minimized total power that is obligatory for cooperation based transmission. In contrast the lesser values of $\mathrm{Pb}$ leads to high power obligatory for the transmission of data in 2 nd stage. This trade-offs stated in certain research papers and in-depth exploration is planned as an approach to find optimum value of broadcast power [13].
According to current paper, an efficient structure is presented for methodically resolve the optimization issue which is addressed above.

\section{FLOW-CHART AND METHODOLOGY OF DIST-COOP}

The proposed look into system is quickly clarified through the accompanying flow chart in Fig. 2. Irregular sending of 250 sensors submerged with 5 sinks on the water surface in a domain of $500 \times 500 \times 500$. Each sensor will begin to find its neighbouring sensors. This will make the sensors to recognize the best option of neighbours which can be chosen for data transfer based on remaining energy and estimated cost function. It is imperative on the grounds that each node which is encompassed by different sensors which is diverse with various sensors because of node thickness. In the focused on territory sensors are scattered arbitrarily.

After estimation of neighbour sensors, in subsequent stage we ascertain the residual energy (RE) of the considerable number of sensors conveyed. Here we separate the sensors on edge energy level 1 and 2 . In the event that the R.E of the node is more prominent or equivalent to edge energy level 1 , at that point this node can be chosen as a transfer node. In the event that the RE is not as much as limit energy level 1, at that point hand-off node again process its $\mathrm{RE}$, if the $\mathrm{RE}$ is more noteworthy than edge level 2 and not as much as edge level 1 , so it can have the capacity to use as a typical sensor node. On the off chance that, if R.E is not as much as edge level 2 than it begins its Energy Harvesting $(\mathrm{EH})$ to increase its energy level.

\section{Dist-Coop Protocol Mathematical Modelling}

In this segment, we present our estimated optimized congestion control and link aware with Cooperation based scheme Dist-Coop which guarantees to enhance the Network Lifetime, Packets Delivery Ratio of the system and lessened Energy use by sensors.

\section{A. Network Topology}

System limit, energy utilization and the unwavering quality of a system relies upon arranged topology. The greatest scope of a sensor node is not sufficient to cover the whole system, so multi-hop correspondence is utilized. Information got from the source node at sink node is accumulated. It might consider that sink node has no energy requirement that may speak with any of the sensors without collaboration. System is isolated into various layers based on profundity and is made out of heterogeneous sensors. The lower profundity sensors send information to higher profundity sensors and the procedure goes ahead till the information comes to at the surface of the water. The transfer sensors are propelled node, since they have double duties of transmitting its own information and also transferring of neighbor sensors. In Fig. 6, if there should be an occurrence of ordinary information transmission the source node transmit information toss two hand-off sensors in a collaboration mode, on the grounds that if hand-off sensors isn't accessible or may dead, so there will be another hand-off and an immediate connection accessible for the information exchange. 


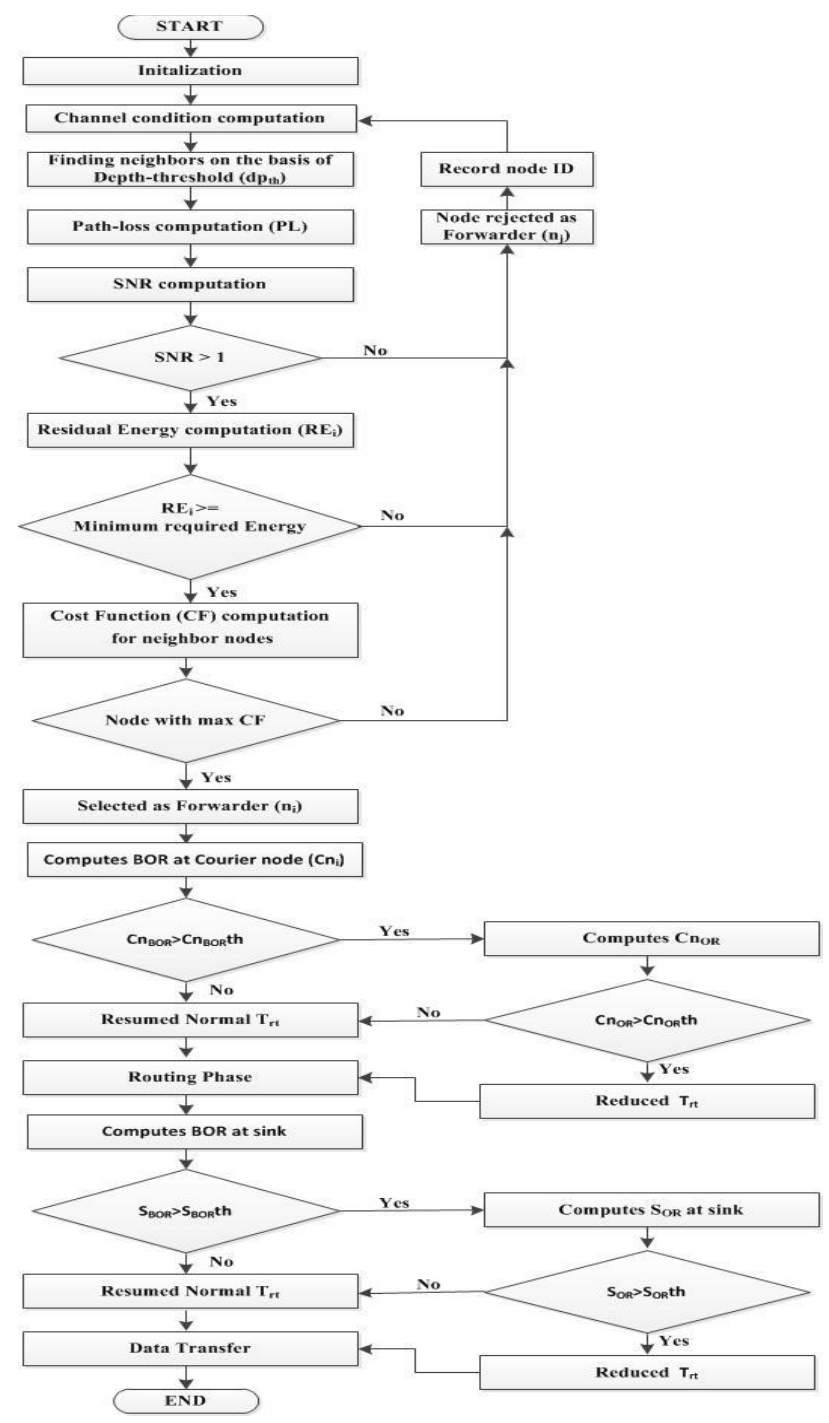

Fig. 2. Flow chart of the Scheme EH-UWSN.

\section{B. Absorption and Scattering Models}

Two noteworthy reasons of constriction in submerged condition are scrambling and ingestion misfortunes. In disseminating, the electromagnetic signals is diverted far from its unique bearing, and in assimilation, the electromagnetic signals energy is changed over starting with one shape then onto the next frame like warmth or substance. Consequently:

$c(\lambda)=a(\lambda)+b(\lambda)$

Where $\mathrm{a}$ and $\mathrm{b}$ refer to ingestion and dissipating separately, estimated in $\mathrm{m}-1$, and $\lambda$ is the wavelength of signals in $\mathrm{nm}$. Utilizing the co-productive of weakening Beer, s Law decide the construction of an acoustic signals for a separation $d$ is given by [25]:

$$
\mathrm{I}=\operatorname{Io} \mathrm{e}^{-\mathrm{c}(\lambda)} \mathrm{d}
$$

Where Io is a normalizing steady. The standardization esteem is a non-negative esteem, relies upon the circumstance to make the change precisely equivalent to $1 . \mathrm{c}(\lambda)$ is coproficient of lessening, $d$ is a separation.

\section{Ambient Noise}

Surrounding commotion is one of the vital factor in submerged acoustic channel condition. Encompassing commotion is fundamentally a connection between the measure of data worried about air state of the sea, ocean condition of the sea, wind speed and sea life natural impacts. Four fundamental sources show the distinctive overwhelming levels of surrounding commotion. They are: Turbulence, waves, delivery and warm clamor. The aggregate power unearthly thickness of clamor is communicated in $\mathrm{db}$ and is given by [27]:

$$
\mathrm{NL}=\mathrm{NLtb}+\mathrm{NLsh}+\mathrm{NLwV}+\mathrm{NLth}
$$

Where

$$
\begin{aligned}
& \text { NLtb }=27-30 \log \mathrm{f} \\
& \text { NLsh }=40+20(\mathrm{~s}-0.5)+26 \log \mathrm{f}-60 \log (\mathrm{f}+0.03) \\
& \text { NLwv }=50+7.5(\mathrm{w}-0.5)+20 \log \mathrm{f}-40 \log (\mathrm{f}+0.4) \\
& \text { NLth }=-25+20 \log \mathrm{f}
\end{aligned}
$$

$\mathrm{f}$ in $\mathrm{KHz}$, w is wind speed $(\mathrm{m} / \mathrm{s})$ and s- shipping activity factor.

\section{Signal-to-Noise Ratio (SNR)}

The SNR of an underwater acoustic signal at a receiver side can be calculated in $\mathrm{dB}$ by sonar equation [27] as follows:

$$
\mathrm{SNR}=\mathrm{SL}-\mathrm{TL}-\mathrm{NL}-\mathrm{DI}+\mathrm{c}(\lambda)
$$

Where

$\mathrm{NL}$ is ambient noise level in ocean $(\mathrm{dB})$,

$\mathrm{TL}$ is transmission loss $(\mathrm{dB})$,

DI is the directivity index and is set to zero and SL is the source level of transmission $(\mathrm{dB})$ is given by

$$
\mathrm{SL}=10 \log \left(\frac{\mathrm{Pit}}{0.67 \times 10(-18)}\right)(\mathrm{dB})
$$

Where Pit is the transmission power intensity.

In shallow water, the Intensity, Pit is given in watt $/ \mathrm{m} 2$ as follows.

$$
\text { Pit }=\frac{\mathrm{Pt}}{2 * \pi * \mathrm{z}}
$$

In deep water, $\mathrm{Pt}$, is given in watt/m2 as follows

$$
\text { Pit }=\frac{\mathrm{Pt}}{4 * \pi * \mathrm{~d}}
$$
(m)

Where, Pt is the transmitted power (watt) and $\mathrm{d}$ is the depth

\section{E. Initialization Phase}

Three unique assignments are performed in this phase. Every sensor has information about its neighbor, sink node on the water surface is recognized, and every conceivable course toward various sink is assessed. Sensors communicate a packet, which contains data of the node like its profundity, node ID, and energy status. This packet has been gotten by the neighbor node and utilizes these for advance transmissions. Sink present on the water surface sends a Hello packet to each 
of its associated sensors. Utilizing high bundle transmission, with in the given transmission extend, every node distinguish its neighbor and independently kept up a line of neighbor under profundity limit to recognize the best sent node for its information transmission; Each node figure its weight utilizing the recipe given beneath [27].

$$
\mathrm{Wi}=
$$

$\max \left(\operatorname{SNR}\left(d_{\mathrm{SiR} 1}, \mathrm{f}\right), \operatorname{SNR}\left(\mathrm{d}_{\mathrm{SiR} 2}, \mathrm{f}\right) \operatorname{SNR}\left(\mathrm{d}_{\mathrm{SiD}}, \mathrm{f}\right)+\max \left(\mathrm{R} \cdot \mathrm{E}_{\mathrm{R} 1}, \mathrm{R} \cdot \mathrm{E}_{\mathrm{R} 2}, \mathrm{R} . \mathrm{Edi}\right)\right.$

(8)

$$
\min \left(\left|\mathrm{d}_{\mathrm{SiR} 1}\right|^{2},\left|\mathrm{~d}_{\mathrm{SiR} 2}\right|^{2},\left|\mathrm{~d}_{\mathrm{SiDi}}\right|^{2}\right)
$$

Where SNR(dSiR1,f), SNR(dSiR2,f), SNR(dSiD1,f) are the SNR of the corresponding links from Si to Di respectively, $\mathrm{R} . \mathrm{E}$ is the residual energy of the corresponding sensors; dSiR1, $\mathrm{dSiR} 2$, and $\mathrm{dSiDi}$ are the distances from the corresponding source to its relays and immediate destination respectively.

\section{F. Underwater Acoustic Cooperative Transmission}

Let $\mathrm{N}$ fixed agents that are sensed and they represented by Si where the $i \in N:=\{1, \ldots, N-1, N\}$. Relay sensor nodes are also arbitrarily set up in $2 \mathrm{dim}$ area. Suppose index of neighbor relays and other nodes for the ith node belongs to sets $\mathrm{Li}$ and Ni. Assume relaying and sensing sensor nodes are capable for adjusting power of transmissions while they all have same maximum power of transmission that is denoted by Pmax. Transmission range of agents that are sensing, directly related to maximum power of transmission. The sensor nodes are known as neighbors if theirs Euclidean distances are minimal than transmission range. In this process the major aim of sensor nodes deployments is observing the geographical area and transfers the data to a static BS. BS is likely as the sink and other nodes are the sources that sporadically measure data.

\section{G. Cooperation based Model of Transmission}

Assortment system stated as a system which takes more than two same replicas of transferred signals from the transmitter. According to this research paper, we deliberate a cooperation based protocol with two relay nodes as Fig. 3. It contains one source node, two relay nodes with a single destination node.

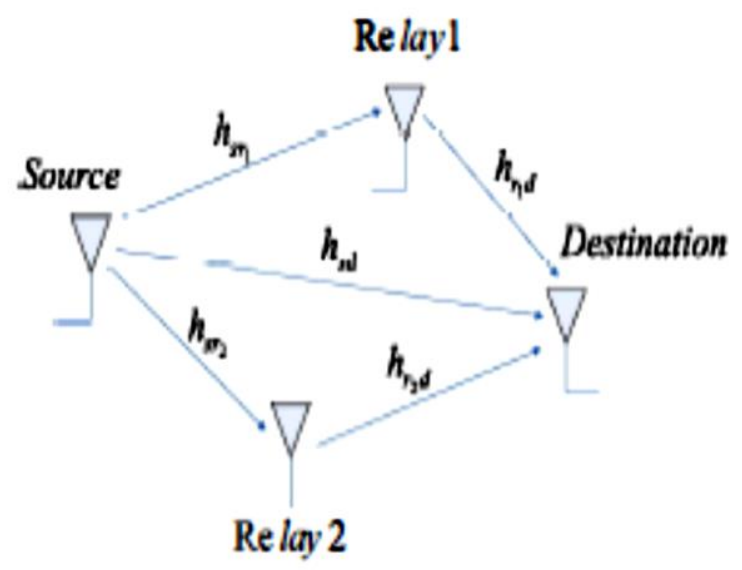

Fig. 3. Cooperative Transmission with Two Relays.
In Fig. 3, relay nodes depend on reformative cooperation while relay nodes sensed and transfers original message that comes from source node prior to forward decrypted bits to terminus [7]. In TWSNs transmission time is lesser while processed using phase synchronization procedures. Due to long transmission delays in UWSNs, addition scheme depends on analogue domain which flops as three signals reach to terminus at different time. Assortment merging methods are auspicious resolutions for processing the receive signals at terminus for UW communications with maximal ratio diversity, equal gain diversity and assortment diversity system [13]. It is observed that assortment methods diminish impacts of declining and increased channel excellence. These methods are mostly model for analogue waves in radio transmission that's propagation delays greatly low as compare to processing delays at all sensor nodes. Though, the data signals travel alongside more than one channel (relays and terminus sensor nodes) with multi extents and arrived to terminus at diverse timings in UW environ. It is not possible to apply addition model to received signals at terminus in term of analogue data signals. Thus, our technique adopts diversity concepts by merging procedures which are applied for analogue field at physical layer to receive data signals at packet level relying on channel state information (CSI).

\section{H. Selection of Relay and Destination}

According to literature [8], [14], a source sensor node tries to search optimal relays to make possible the cooperative communications for TWNs with supposition the destination is pre-defined. This methodology depends on terminated timers at the possible relay nodes, is unsuitable for UW links as it unusually maximizes delays of UW channels. Moreover, link states possibly modify by high delays due to terminated timers or collision evasion amongst devices. This research examines cooperative base communications by distributed mode for UWSNs. In this paper it is assumed that a source sensor node, that requires transferring their message to sink node by group of hops, have $\mathrm{n}$ neighboring sensor nodes in its range of transmission that is shown in Fig. 4. Source sensor nodes rely on instant link state for determining that which ones amongst neighbors have maximum consistent links to transfer the information to sink node containing the relay and destination nodes. Channel properties are taken in account by electing processes. It contains SNR from all links to source sensor node and distance node from all neighbors to sink node. It is important to note that GPS systems that are fortified for terrestrial wireless based devices not able to work fine in case of the environments of UW by the restrictions of link properties as well as frequencies. Transmission processes consist of a sequence of six phases that are:

- Request to Send (RTS)

- Clear to Send (CTS)

- Source based computations

- Source transmissions

- Relays transmissions

- Acknowledgement. 


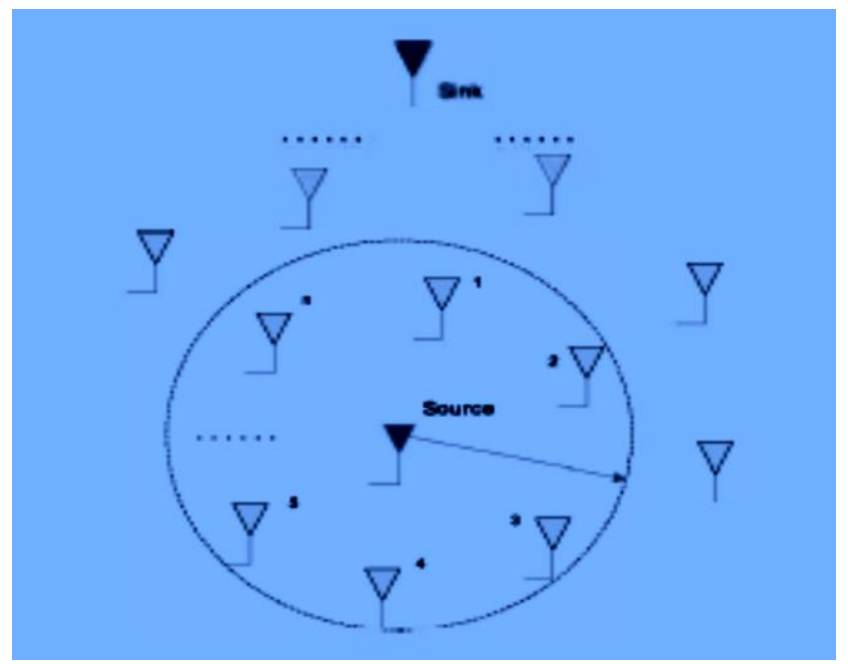

Fig. 4. Network model with source and neighbors at each hop.

\section{Creating Neighbors List}

A list of neighbor sensor nodes are created by every sensor node with particular hop-count that is known as distance interchangeably to sink node by advertising (ADV) data packet that sporadically transmits from sink node to each node afterward every pre-defined time period. Depending on hopcount to sink node, source node just transmits the data packet to sink node by a set of relative neighbors. After initiative time, the network state is constant; and ADV data packet shows part of altering link state among local sensor nodes and neighboring nodes.

\section{J. Collecting Condition of Link \Channel}

Initially in case that a source sensor node has data then this node broadcast RTS packet towards neighboring nodes that potential relay nodes and destination to relays packets. The size of a RTS and CTS is lesser then data packet's for reducing the consumption of energy for neighboring sensor nodes. A RTS packet consists of hop count of source sensor node to sink node. As a neighboring sensor node gets RTS packet, it must compare their local hop-count to sink node with hop-count of source sensor node. Just neighboring nodes having shorter distance than source sensor node, possibly become next relay. It restricts number of sensor nodes to join in to rivalry to become destination/ relays to decrease the consumptions of energy and packet loops. After inspection of distance, potential relay replies a CTS packet to source node. Note that according to this technique, a sensor node impossible measure channel status info between itself and destination node such as [8], [14]. It is by ambivalent to the destination node. Though, link state from relay node to destination node is a significant parameter that effects by the selection of relay node. Hence all sensor nodes sensed neighboring link state in terms SNR by eavesdropping data packets from their neighbors and inset average value in CTS.

In case of distributed scenario, more than one source nodes needed to forward data packet, CTS from all neighbors recognizes to strike on links. However, data sensed rate from environ measures for all sensor nodes supposed to minor to decrease data packets collisions. Source sensor nodes estimate
Time of Arrival (ToA) and SNR on the reception of CTS packet conforming to particular neighbors. ToA is a packet that travels from a sensor node to other. That parameter is for the measuring physical distance from a source node to a potential relay and terminus. SNR kept conforming to a neighbor that is be around between sensed SNR from CTS at source node while be an average of SNR which is approximate by eavesdropping at every neighbor.

\section{K. Destination/Relays Selection}

After the RTS/CTS exchange procedure, the source node achieves a list of candidates with the distance, ToA, and channel conditions, respectively. The source runs Algorithm 1 to select the appropriate and reliable relay nodes to forward the data message.

Algorithm 1: Destination/Relays Selection.

Input : SNR, Hop_count, ToA by parents Childs with siblings

Output: Destination with two relay nodes

$$
\begin{aligned}
& \max :=\text { total neighbours }(\mathrm{n}) \text { with } \mathrm{d}(\mathrm{x}) \geq \mathrm{d}(\mathrm{n}) \text {; } \\
& \text { for } \mathrm{x}=1 \text { to } \max
\end{aligned}
$$$$
\text { If } \mathrm{C}(\mathrm{f}, \mathrm{l}) \geq \mathrm{R} \text { then }
$$$$
\text { Requirement in Eq10 }
$$

If $\mathrm{d}(\mathrm{x})>\mathrm{d}(\mathrm{n})$ then

Add with sort using ToA in parents list; else

Add with sort using ToA in siblings list;

$$
/ / \text { where } d(x)=d(n)
$$

Select (top - down) three members from parents list with siblings for destination and two relay nodes;

The siblings and parents are neighbors (n) whom hop-count are lesser and equal to source ( $\mathrm{x}$ ) on link to sink node. Neighbors with hop count lower or equal to the source sensor nodes combined into relays computation. The three parameters used for evaluating a contender with SNR, ToA and hop-count. Initially source node examines computed link capacity correspond to neighbors and requisite rate of data included in candidates list. Afterward the algorithm examines that sensor node is parent or sibling, hence then it includes to particular lists as well as sort by ToA. The timer is also set for getting CTS packet; information of neighbors updates and stops if the timeout happens. Till each candidate is tested or timeout, source sensor node selects three members to be a terminus as well as two relay nodes from two that lists. Source node can select destination or relay node soon on ending of every list for flexibility.

\section{Techniques for Diversity Combining}

The UW links faces a quite great propagation delays that becomes the reasons of great difference for arriving data signals. Links with source nodes, relay nodes and the destination nodes are greatly lengthier as compared to direct source node to destination route. Therefore the techniques of 
diversity combining are appropriate choice for processing the signals at destination node, while incoming signals from the source node and relay nodes are operated at packet level, instead of at physical layer. In 1st phase, intermediary relay nodes translates message that comes from a source node and transfer it to next. Destination node then takes one or more copies from the source node and relay nodes. The technique called maximal ratio combing (MRC) is then applied for recovering the messages from source node as well as intermediary relay nodes. We used BER estimation model to compute BER of data packets that's parameters assessed by the technique named maximum likely-hood estimation (MLE).

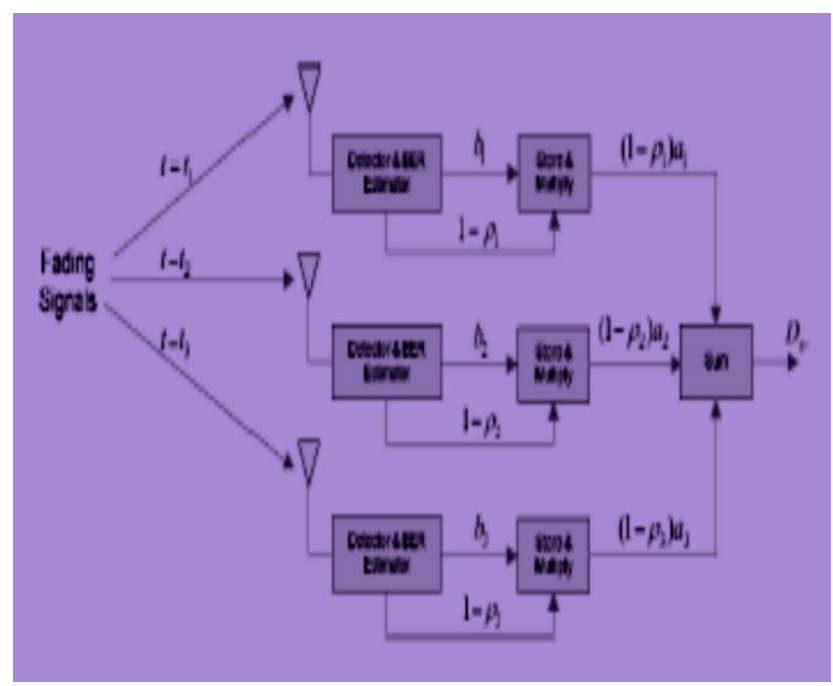

Fig. 5. The maximal ratio combining.

MRC is shown in Fig. 5 by a diagram for Multiple Input and Single Output (MISO) with three inputs. We also used three incoming routes at $\mathrm{t} 1 ; \mathrm{t} 2$ and $\mathrm{t} 3$ times that are from source node, 1 st relay node and 2 nd relay node. At destination node, arrived signals from link are de-modulated and translated to get a binary result that is bi where $i=1,2$ and 3 . Sensed binary sequence is retained and multiplied by the respective weight factors that are 1- Pi for the recovery of original message from various copies. Moreover the bits are transformed to biased value that is $a_{i}$ prior to multiply, where

$$
a_{i}=\left\{\begin{aligned}
1 & \text { for binary } 1 \\
-1 & \text { for binary } 0
\end{aligned}\right.
$$

The output binary value $b$ is determined by the sum Do based on the threshold which is set to zero.

$$
\begin{aligned}
& \mathrm{D}_{0}=\sum_{\mathrm{i}=1}^{3}\left(1-\mathrm{p}_{\mathrm{i}}\right) \cdot \mathrm{a}_{\mathrm{i}}, \\
& \mathrm{b}= \begin{cases}1 & \mathrm{D}_{0} \geq 0 \\
0 & \mathrm{D}_{0} \text { i } 0\end{cases}
\end{aligned}
$$

\section{Routing Model}

A K-hop cooperative route ' is a sequence of $\mathrm{K}$ links $\left\{l_{1} \ldots \ldots \ldots l_{k}\right\}$, where each link $l_{k}=\left\langle t_{k}, r_{k}\right\rangle$ is formed between a transmitting node $t_{k}$ and a receiving node $r_{k}$, using the two-stage cooperative transmission at the physical layer. The sequence of links $l_{k}$ connects a source ' $\mathrm{s}$ ' to a destination ' $d$ ' in a loop-free path. Our objective is to find a path that minimizes end-to-end transmission power to reach the destination.

Cost of Link: The link cost $l_{k}=\left\langle t_{k}, r_{k}\right\rangle$ that is represented by following

$\mathrm{C}=\left(\mathrm{t}_{\mathrm{k}}, \mathrm{r}_{\mathrm{k}}\right)$

The above equation can be stated as least expected power of transmission for delivering a message from $t_{k}$ to $r_{k}$ by a cooperative transmission that consist of two-stage system that is subject to rate $\lambda$ with outage probability which is denoted by $\mathrm{p}_{\mathrm{e}}$. Then, the problem of energy efficient routing can be formulated as follows:

$$
\min _{\mathrm{l}_{\mathrm{k}} \in \mathrm{L}} \sum_{\mathrm{l}_{\mathrm{k} \in \mathrm{l}}} \mathrm{C}\left(\mathrm{t}_{\mathrm{k}}, \mathrm{r}_{\mathrm{k}}\right)
$$

In above equation the $\mathrm{L}$ represents set of entire possible links in network (any free of loop sequence of sensor nodes from source node to destination node is a possible path of this model).

\section{N. Optimal Cooperation-based Routing}

1) Link Cost Formulation: Let we have a link $\left\langle\mathrm{t}_{\mathrm{k}}, \mathrm{r}_{\mathrm{k}}\right\rangle$ that is designed between two sensor nodes that are $t_{k}$ and $r_{k}$ by two-stage cooperation based transmission. The $t_{k}$ is the set of cooperative sensor nodes in 2nd stage transmission approach. The power allocation vector to form the cooperative link $\left\langle t_{k}, r_{k}\right\rangle$ is represented by $p$. Expected cost of cooperative link $\left\langle t_{k}, r_{k}\right\rangle$ represented by $C\left(t_{k}, r_{k}\right)$ that is given by following 6.5 optimization issue,

$$
C\left(t_{k}, r_{k}\right)=\min _{p \in P} \frac{\sum_{t_{i} \in T_{k}} p_{i}}{S\left(T_{k}, p, r_{k}\right)}
$$

The $\mathrm{P}$ in above equation represents set of all possible power allocation vectors denoted by $p$, where $P_{\max } \geq p_{i}$ is power that allocated to transmitter $t_{i} \in T_{k}$.

The major advantage of cooperation based transmissions is in fading environs in which diversity is used for conflict fading. Applications which take advantage from cooperation based transmissions are usually have a severe obligation in terms of reliability of link. Link cost construction is addressed in (5). Though, it not give any particular target outage probability and no check on the number of re-transmissions with consequently link delay. To control this problem, we alter optimization 5 to comprise a restriction on target outage probability as following.

Suppose $\mathrm{p}_{\epsilon}$ represent target per link outage probability which can be accepted. Then cooperative link cost $C\left(\mathrm{~T}_{\mathrm{k}}, \mathrm{r}_{\mathrm{k}}\right)$ is the solution to the following constrained optimization problem:

$$
C\left(t_{k}, r_{k}\right)=\min _{p \in P} \sum_{t_{i} \in T_{k}} p_{i}
$$

Subject to $S\left(T_{k}, p, r_{k}\right) \geq 1-p_{\epsilon}$

Till this point, entire cost of transmission to create link $\left\langle t_{k}, r_{k}\right\rangle$ is sum of transmissions power in 1 st and 2 nd stage. It is expressed by following

Total Power to create $\left\langle\mathrm{t}_{\mathrm{k}}, \mathrm{r}_{\mathrm{k}}\right\rangle=\mathrm{C}\left(\mathrm{T}_{\mathrm{k}}, \mathrm{r}_{\mathrm{k}}\right)+\mathrm{P}_{\mathrm{b}}$ 
The $\mathrm{T}_{\mathrm{k}}$ is cooperation based transferring set that is created in 1 st stage.

2) Least Cost Route Selection: Now we model the network as weighted graph that is denoted as following:

$\mathrm{G}=(\mathrm{N}, \mathrm{E}, \mathrm{C})$,

Where $\mathrm{N}$ is set of sensors of the network, $\mathrm{E}$ is set of entire conceivable links in sensor nodes that are given below:

$$
\begin{aligned}
& E=\left\{\left(t_{k}, r_{k}\right) \mid t_{k}, r_{k} \in N\right\} \text { and } \\
& C=\left\{C\left(t_{k}, r_{k}\right) \mid\left(t_{k}, r_{k}\right) \in E\right\}
\end{aligned}
$$

It is set of costs of link that is well-defined over ends. The issue of efficient energy routing now expressed as shortest path problem on $G$ where $G$ is the graph. By Use of Dijkstra's algorithm, least route in source node and destination node calculated by $\mathrm{O}(\mathrm{N} \log \mathrm{N})$, in case if costs of link $\mathrm{C}$ is known. Though $\mathrm{C}$ is calculated one time and it is possible to calculate offline. The calculating link costs comprise enumerating the exponential amount of cooperative set called T. To ease it, a strategy is reducing search space for $\mathrm{T}$ that is addressed in next section.

\section{O. Opportunistic Route}

Due of anycast technique, the messages arrive at destination by possibly different paths. A devious path is amalgamation of multiple conceivable paths within the source node and the destination node that is created by a choice of candidate relay nodes at every intermediary sensor node.

1) Anycasted Link Cost: Deliberate a transmitter denoted by $t_{k}$ with the corresponding candidate relay node set $R_{k}$. In phase $1, t_{k}$ broadcast a message with power $\mathrm{Pb}$. The sensor nodes which positively took message join $t_{k}$ to form $a$ cooperation based communicating set $\mathrm{T}_{\mathrm{k}}$. In phase $2, \mathrm{~T}_{\mathrm{k}}$ cooperatively anycasted message to entrant relays set $\mathrm{R}_{\mathrm{k}}$.

2) Anycast Link Cost: Anycasted link cost $\mathrm{l}_{\mathrm{k}}=\left\langle\mathrm{t}_{\mathrm{k}}, \mathrm{R}_{\mathrm{k}}\right\rangle$ is represented by $C\left(t_{k}, R_{k}\right)$ which can be stated as least expected power of transmission to send message from $t_{k}$ to any sensor node in $\mathrm{R}_{\mathrm{k}}$ by using two stage cooperation based transmission subject to rate $\lambda$ with outage probability which is denoted by $p_{\epsilon}$. Suppose $C\left(T_{k}, R_{k}\right)$ represent least power that is requisite for cooperation based anycasting from $T_{k}$ to $R_{k}$. Hence $C\left(T_{k}, R_{k}\right)$ is expressed by following optimization issue:

$$
\mathrm{C}\left(\mathrm{T}_{\mathrm{k}}, \mathrm{R}_{\mathrm{k}}\right)=\min _{\mathrm{p} \in \mathrm{P}} \sum_{\mathrm{t}_{\mathrm{i}} \in \mathrm{T}_{\mathrm{k}}} \mathrm{p}_{\mathrm{i}}
$$

Subject to $A\left(T_{k}, p, R_{k}\right) \geq 1-p_{\epsilon}$

Where $A\left(T_{k}, p, R_{k}\right)$ represents probability which at least one sensor node in set of $\mathrm{R}_{\mathrm{k}}$ positively received messages that is uttered as

$$
A\left(T_{k}, p, R_{k}\right)=1-\prod_{r_{j} \in R_{k}}\left(1-S\left(T_{k}, p, r_{j}\right)\right)
$$

Using (18), anycasted cost of link $C\left(t_{k}, R_{k}\right)$ is expressed by following optimization issue on broadcast power that is denoted by $\mathrm{P}_{\mathrm{b}}$ :

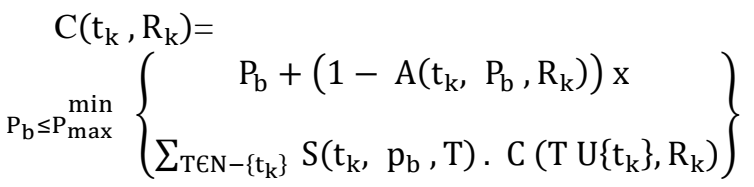

Where $S\left(t_{k}, p_{b}, T\right)$ is given by $(15)$, and $A\left(t_{k}, P_{b}, R_{k}\right)$ can be computed from (18) by substituting $\mathrm{T}_{\mathrm{k}}=\left\{\mathrm{t}_{\mathrm{k}}\right\}$

\section{P. Optimization Parameters}

The variables of decision for the issue of global optimization are broadcast power at source, cooperation based transmission power for relay node and sources. For different single hop transmission, certain relay nodes might be engaged by different sources at different timing. Hence different the values of cooperation based transmission power are determined. Assume that ith source contains ni $=\operatorname{dim}(\mathrm{Ni})$ neighbor relays, and represent the values of cooperation based transmission power by $\mathrm{Pk}, \mathrm{i}$, for any $\mathrm{k} \in\{0,1, \ldots$, ni $\}$. Hence entire transmission power for ith sensor node is entire transmission power of sensor node in broadcast and cooperative transmissions stages, i.e.:

$$
\mathrm{P}_{\text {coop }, \mathrm{i}}:=\mathrm{P}_{\mathrm{b}, \mathrm{i}}+\sum_{\mathrm{k}=0}^{\mathrm{n}_{\mathrm{i}}} \mathrm{P}_{\mathrm{k}, \mathrm{i}}
$$

Note that $\mathrm{P}_{\mathrm{b}, \mathrm{i}}$ is assigned power for source in broadcast stage and $\mathrm{P}_{0, \mathrm{i}}$ is cooperation based transmission power for this sensor node in 2 nd stage. The values of transmission power must accomplish power constraint at sources, relays and SNR restrictions at relays and at corresponding destination for every source. Set of restrictions for cooperation based transmission from ith source to their destination is represented by

$$
\mathrm{C}_{\mathrm{i}}\left(\mathrm{P}_{\mathrm{b}, \mathrm{i}},\left\{\mathrm{P}_{\mathrm{k}, \mathrm{i}}\right\}_{\mathrm{k}=0}^{\mathrm{n}_{\mathrm{i}}}\right) \leq 0
$$

Following stated lemma demonstrate the underlying global optimization issue which is possibly decomposed in to local issues.

Lemma : Let take issue of minimize the entire cooperation based transmission power for sensor nodes as below,

$$
\begin{aligned}
& \min . \sum_{\mathrm{k}=0}^{\mathrm{n}_{\mathrm{i}}}\left[\mathrm{P}_{\mathrm{b}, \mathrm{i}}+\sum_{\mathrm{k}=0}^{\mathrm{n}_{\mathrm{i}}} \mathrm{P}_{\mathrm{k}, \mathrm{i}}\right] \\
& \text { subject to } \mathrm{C}_{\mathrm{i}}\left(\mathrm{P}_{\mathrm{b}, \mathrm{i}},\left\{\mathrm{P}_{\mathrm{k}, \mathrm{i}}\right\}_{\mathrm{k}}^{\mathrm{n}_{\mathrm{i}}}\right) \leq 0, \mathrm{i} \in\{1, \ldots \ldots, \mathrm{N}\}
\end{aligned}
$$

The resolution of above can be acquired by resolving given below $\mathrm{N}$ individual constrain optimization issues for $\mathrm{i}=1, \ldots$. $\mathrm{N}-1, \mathrm{~N}$,

$$
\begin{aligned}
& \min \left[\mathrm{P}_{\mathrm{b}, \mathrm{i}}+\sum_{\mathrm{k}=0}^{\mathrm{n}_{\mathrm{i}}} \mathrm{P}_{\mathrm{k}, \mathrm{i}}\right] \\
& \text { subject to } \mathrm{C}_{\mathrm{i}}\left(\mathrm{P}_{\mathrm{b}, \mathrm{i},},\left\{\mathrm{P}_{\mathrm{k}, \mathrm{i}}\right\}_{\mathrm{k}=0}^{\mathrm{n}_{\mathrm{i}}}\right) \leq 0
\end{aligned}
$$

Proof: Let the set of values $\mathrm{P}_{\text {coop, } \mathrm{i}}^{*}\left\{\mathrm{P}_{\text {coop }, \mathrm{i}}^{*},\left\{\mathrm{P}_{\mathrm{k}, \mathrm{i}}^{*}\right\}_{\mathrm{k}} \mathrm{n}_{\mathrm{i}}{ }_{0}\right\}$

for $\mathrm{i}=1, \ldots \mathrm{N}-1, \mathrm{~N}$, is optimum solution to set of optimization issues (3). The values create a viable solution to optimization issue (2). Variables that are related to ith source not in constraint set for other sources. For proving suitability of solution $\left\{\mathrm{P}_{\mathrm{b}, \mathrm{i}}^{*},\left\{\mathrm{P}_{\mathrm{k}, \mathrm{i}}^{*}\right\}_{\mathrm{k}} \mathrm{n}_{\mathrm{i}}{ }_{0}\right\}$ by illogicality and to be supposed that following is a different possible solution 
$\mathrm{P}_{\text {coop,i }}^{\prime}$ for $\mathrm{i}=1, \ldots \mathrm{N}-1, \mathrm{~N}$ with corresponding $\left\{\mathrm{P}_{\mathrm{b}, \mathrm{i}}^{\prime}\right.$ ,$\left.\left\{\mathrm{P}_{\mathrm{k}, \mathrm{i}}^{\prime}\right\}_{\mathrm{k}=0}^{\mathrm{n}_{\mathrm{i}}}\right\}$ values that results in a improved performance index for (2).

It means that there is an index $\overline{1}$ for which $\mathrm{P}_{\text {coop, } \overline{1}}^{\prime}<\mathrm{P}_{\text {coop, } \overline{1}}^{*}$ or equally $\mathrm{P}_{\mathrm{b}, \overline{\mathrm{I}}}^{\prime}<\mathrm{P}_{\mathrm{b}, \overline{\mathrm{I}}}^{*}$ or $\mathrm{P}_{\mathrm{k}, \overline{\mathrm{I}}}^{\prime}<\mathrm{P}_{\mathrm{k}, \overline{\mathrm{I}}}^{*}$ for $\mathrm{k} \in \mathrm{n}_{\overline{\mathrm{l}}}$. As values $\left\{\mathrm{P}_{\mathrm{b}, \overline{\mathrm{l}}}^{\prime}\right.$ $\left.\left\{\mathrm{P}_{\mathrm{k}, \overline{\mathrm{l}}}^{\prime}\right\}_{\mathrm{k}}=0\right\}$ create a possible solution to $\overline{\mathrm{l}}$-th optimization problem in (3). This produce a improved value for individual optimization issue and this denies optimality of $\left\{\mathrm{P}_{\mathrm{b}, \overline{\mathrm{i}}}^{*}\right.$ ,$\left.\left\{\mathrm{P}_{\mathrm{k}, \mathrm{i}}^{*}\right\}_{\mathrm{k}=0} \mathrm{n}_{\mathrm{i}}\right\}$.

In consequence, the issue of minimum power cooperation based transmission is expressed for a source. Subscript i is released henceforth for ease. The effective tackles of mathematical modelling systems are then engaged to convert underlying optimization issue in to mixed integer linear programming issue.

\section{Simulations, Results AND Discussion}

The proposed protocol Dist-Coop UWSN's performance and effectiveness is evaluated by comparing it with EH-UWSN and SPARCO protocols. 225 nodes having node energy of 0.7 $\mathrm{J}$ were randomly placed in underwater and network dimensions used were $500 \mathrm{~m} \times 500 \mathrm{~m} \times 500 \mathrm{~m}$. The protocol was executed for a total of 100 rounds, having average radius value of $100 \mathrm{~m}$. Network parameters used in simulation are given below in Table I.

TABLE I. NETWORK PARAMETERS

\begin{tabular}{|l|l|}
\hline Parameters & Values \\
\hline Network Volume & $500 \mathrm{~m}$ x 500m x 500m \\
\hline Total Nodes & 225 \\
\hline Relay Nodes & 10 \\
\hline Initial Node Energy & $0.7 \mathrm{~J}$ \\
\hline Number of Rounds & 100 \\
\hline Average Radius value of sensing & $100 \mathrm{~m}$ \\
\hline Number of Sinks & 5 \\
\hline Sensor Node Activation Energy & $6.2500 \mathrm{e}-06$ \\
\hline Amplifying Energy (amp) & $5.0000 \mathrm{e}-09$ \\
\hline Transmitting Energy (Et) & $3.0000 \mathrm{e}-06$ \\
\hline
\end{tabular}

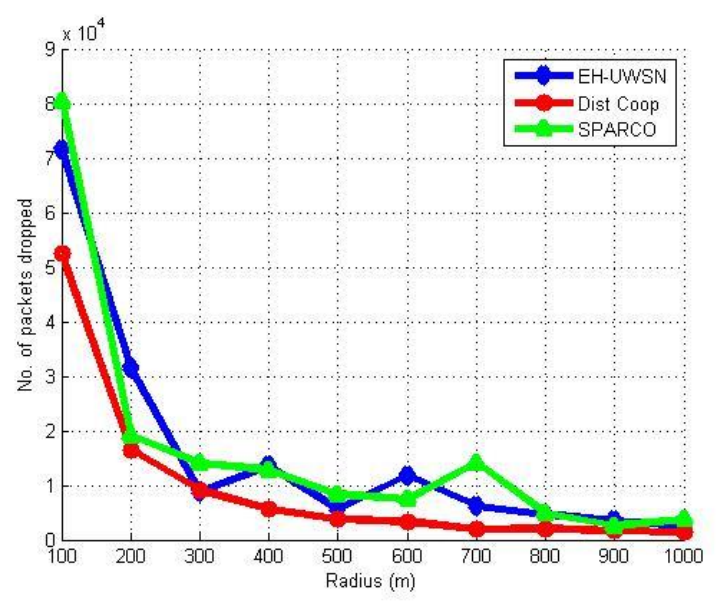

Fig. 6. Number of dropped packets v/s radius (m).

\section{A. Numbers of Dropped Packet}

Fig. 6 shows a comparative analysis of packets dropped at the sink while transmitting sufficient data towards the destination in three schemes. Our presented scheme Dist-Coop is compared with two existing schemes SPARCO and EHUWSN. As our scheme is mainly concentrating on the link reliability and congestion control through the use of cooperation and anycasting strategies, hence Fig. 9 proved that drop of packets in Dist-Coop scheme is much lower as compared to SPARCO and EH-UWSN techniques. Dist-Coop scheme assures that one of the sinks is selected at one time so that data is transmitted in queues and no congestion occurs at the destination sinks. Also opportunist routing enables data to select those paths where path loss is less and link is stable and reliable. Average loss in packets in case of Dist-Coop is 110 while SPARCO and EH-UWSN have 121 and 124 respectively.

\section{B. Energy Tax}

The energy tax level of Dist Coop protocol in Fig. 7 is better than the compared schemes. The average energy consumption of Dist Coop in the figure is near to the energy consumption of SPARCO and EH-UWSN as these schemes mainly focused on the decreasing of energy consumption. Our focused in Dist Coop was on congestion control, increasing stability period and throughput as well as load balancing of motes. Therefore the goal is achieved by showing effective outcomes in these parameters. The Dist Coop is also showing improvement in energy consumption as compared to SPARCO and EH-UWSN.

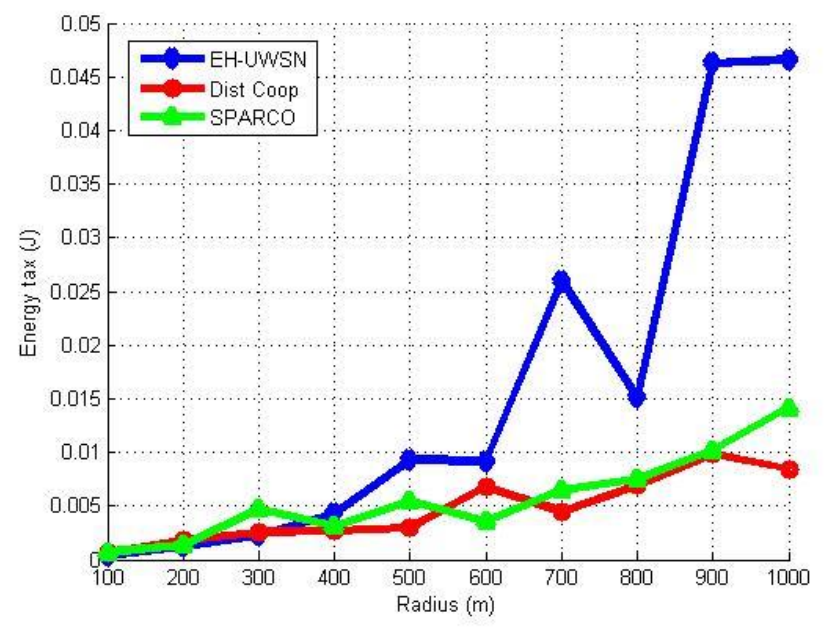

Fig. 7. Energy tax v/s radius (m).

\section{Number of Packets Received}

The concept here is to deliver data effectively from nodes to surface sink. It is also called Packet Delivery Ratio (PDR). Two types of links used in Dist Coop are from sensor node to forwarder and from forwarder to surface sink. Latter one transfers maximum data than the first one. Therefore, in Dist Coop mostly sensors come close to the surface sink and transfer data packets directly which improves the PDR. Fig. 8 shows major efficiency difference between Dist Coop and SPARCO, EH-UWSN schemes. 


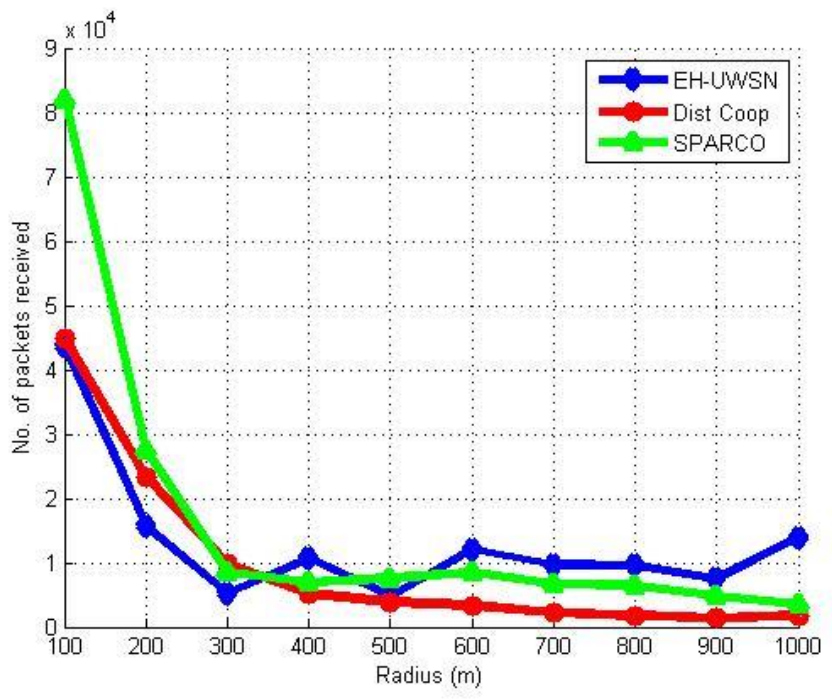

Fig. 8. Received packets v/s radius (m).

\section{Network Stability Period}

Fig. 9 depicts the performance of Dist Coop protocol which is optimal as compared to SPARCO and EH-UWSN techniques. Stability Period is an operational time of the network until 1st mote expires. At seconds 10000 after network starts, Dist Coop loses 102 nodes and 123 nodes are alive while SPARCO and EH-UWSN lose 140 and 178 nodes respectively. This shows that due to balanced usage of energy by the nodes the performance of Dist Coop is improved.

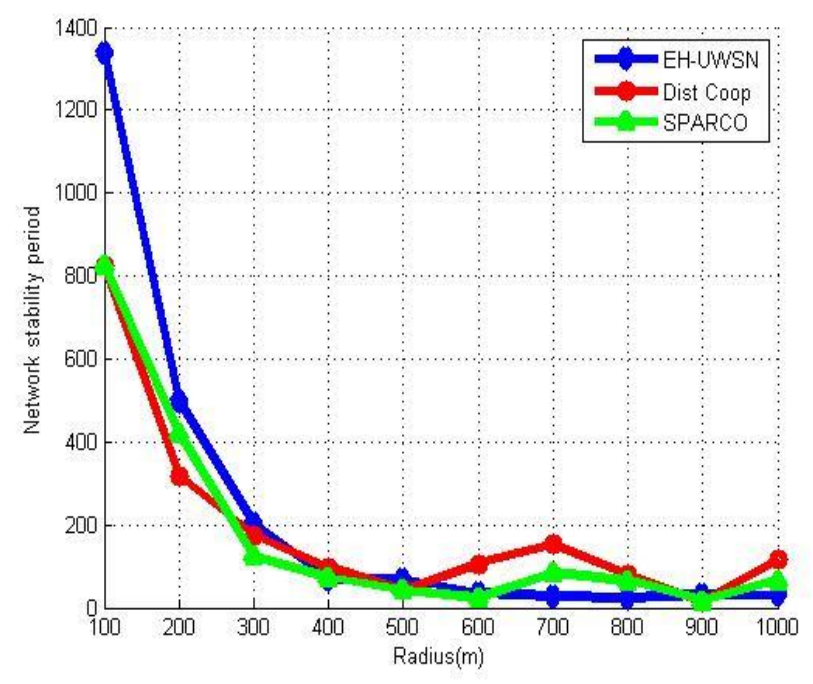

Fig. 9. Network stability period v/s radius (m).

\section{E. Network Lifetime}

Stability Period is an operational time of the network until 1st mote expires. Fig. 10 depicts the optimal performance of Dist Coop protocol as compared to SPARCO and EH-UWSN techniques. After 12000 seconds Dist-Coop lost 93 nodes and 132 nodes are alive while SPARCO and EH-UWSN loses 104 and 115 nodes respectively. This better performance of DistCoop is due to balanced usage of energy by the nodes.

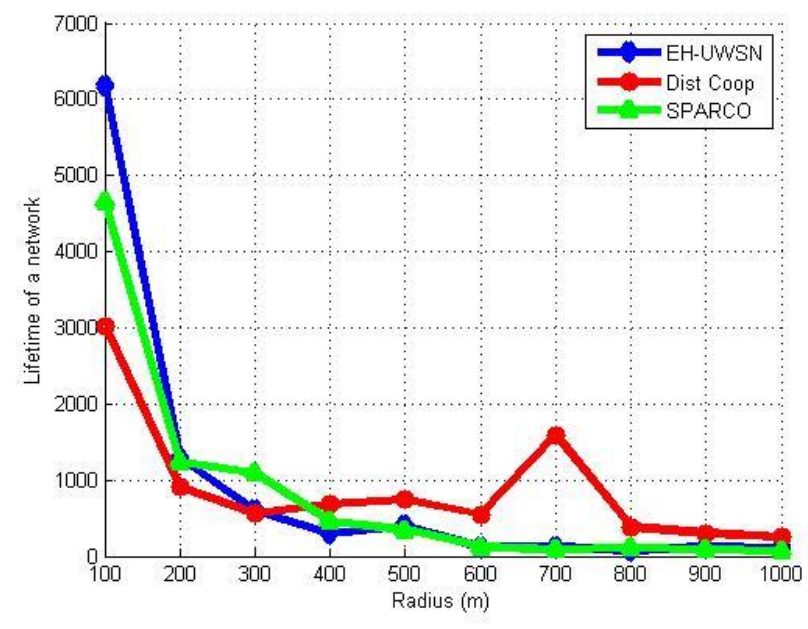

Fig. 10. Network lifetime v/s radius (m).

\section{CONCLUSION AND FUTURE WORK}

In this research, we have presented a congestion control scheme whose focus is on the network stability period extension and link awareness called Dist-Coop to tackle the aforementioned issues of UWSNs upto some extent. After the analysis of simulation results, we conclude that Dist-Coop showed extraordinarily better execution in connection than the existing schemes i.e. SPARCO and EH-UWSN as far as system Lifetime, Packets Delivery Ratio, Energy Consumption, Packets Received Ratio and Stability Period of the system are concerned.

Dist-Coop has devoured substantially less energy up to 3 times when contrasted with SPARCO and EH-UWSN amid the transmission of information. In Fig. 7, at first from sweep $100(\mathrm{~m})$ to $500(\mathrm{~m})$ the two plans have expended roughly level with energy, however after 500(m) the SPARCO consumed much energy when contrasted with EH-UWSN protocol. Huge contrast is appeared in the normal estimations of Dist-Coop and EH-UWSN plans. In Dist-Coop plot, sooner or later where energy is collected, than little energy is devoured in that segment of the plot like 500(m) to 600(m).

Furthermore, Dist-Coop improved the system lifetime by gathering energy from the earth to make a sensor alive for longer time. In Fig. 8, the underlying life time organize estimation of SPARCO from radius $100(\mathrm{~m})$ to $400(\mathrm{~m})$ is substantially more prominent when contrasted with EH-UWSN protocol. At sweep $400(\mathrm{~m})$, both of the plans have risen to values around. On the off chance that we analyze the plot of EH-UWSN protocol, it plainly demonstrates that its system life time esteems rot fastly when range increments from $100(\mathrm{~m})$ to 1000 (m). The EH-UWSN esteem bit by bit diminishes when the sweep increments, while plot of Dist-Coop protocol demonstrates energy collecting at various focuses in Fig. 9. At the point when sweep is equivalent to $500(\mathrm{~m})$, Than Dist-Coop protocol has little system life time esteem, so it begins collecting energy up to some level from span 500(m) to 600(m) with a specific end goal to expand the life time of a system. Energy gathering is rehashed as appeared in Fig. 10, when the energy level reductions from the characterize limit esteem. The reproduction comes about shows Improvement in Stability time 
of the Network in Dist-Coop amid the examination with existing plan SPARCO in Fig. 10. The normal estimation of Dist-Coop protocol is $106 \%$ enhanced when contrasted with UWSN existing schemes. As the energy productivity and dependability of a system relies upon the parameters under thought, henceforth clearly Dist-Coop is more energy proficient, link aware and congestion controlled than SPARCO and EH-UWSN schemes.

In our scheme, we utilized SNR consolidating methodology (SNRC) at destination to join the signals received from various directions. In future, we can utilize distinctive different methodologies like, Fixed Ratio Combining (FRC), Maximal Ratio Combining (MRC) and Selection Combining (SC) at destination to inspect and contrast the results and the introduced strategies so as to outline more productive routing schemes for various UWSNs and WSNs environments. By utilizing this method, researchers can additionally enhance system life time by growing further developed protocols.

\section{REFERENCES}

[1] Kaur, and S. Monga, "Comparisons of wired and wireless networks: A review." International Journal of Advanced Engineering Technology 5, no. 2 (2014): 34-35.

[2] Frodigh, P. Johansson, and P. Larsson. "Wireless ad hoc networking: the art of networking without a network." Ericsson review 4, no. 4 (2000): 249.

[3] Penttinen, "Research on ad hoc networking: Current activity and future directions." Networking Laboratory, Helsinki University of Technology, Finland. See also http://citeseer. nj. necm. com/533517. html (2002).

[4] Wahid and K. Dongkyun, "Analyzing routing protocols for underwater wireless sensor networks." International Journal of Communication Networks and Information Security 2, no. 3 (2010): 253.

[5] Heidemann, W. Ye, J. Wills, A. Syed and Y. Li, "Research challenges and applications for underwater sensor networking." In Wireless Communications and Networking Conference, 2006. WCNC 2006. IEEE, vol. 1, pp. 228-235. IEEE, 2006.

[6] Heidemann, M. Stojanovic and M. Zorzi. "Underwater sensor networks: applications, advances and challenges." Phil. Trans. R. Soc. A 370, no. 1958 (2012): 158-175

[7] Wahid and D. Kim, "An energy efficient localization-free routing protocol for underwater wireless sensor networks." International journal of distributed sensor networks 8, no. 4 (2012): 307246.

[8] S. Ahmed, I. U. Khan, M. B. Rasheed, M. Ilahi, R. D. Khan, S. H. Bouk and N. Javaid,"Comparative analysis of routing protocols for under water wireless sensor networks." arXiv preprint arXiv:1306.1148(2013).

[9] M.R. Jafri, S. Ahmed, N. Javaid, Z. Ahmad and R. J. Qureshi, "Amctd: Adaptive mobility of courier sensors in threshold-optimized dbr protocol for underwater wireless sensor networks." In Broadband and Wireless Computing, Communication and Applications (BWCCA), 2013 Eighth International Conference on, pp. 93-99. IEEE, 2013.

[10] Javaid, M. R. Jafri, Z. A. Khan, U. Qasim, T. A. Alghamdi and M. Ali, "Iamctd: Improved adaptive mobility of courier sensors in thresholdoptimized $\mathrm{dbr}$ protocol for underwater wireless sensor networks." International Journal of Distributed Sensor Networks 10, no. 11 (2014): 213012.

[11] Javaid, M. R. Jafri, S. Ahmed, M. Jamil, Z. A. Khan, U. Qasim and S. S. Al-Saleh, "Delay-sensitive routing schemes for underwater acoustic sensor networks." International Journal of Distributed Sensor Networks 11, no. 3 (2015): 532676.
[12] A. Khan, N. Javaid, A. Majid, M. Imran and M. Alnuem, "Dual sink efficient balanced energy technique for underwater acoustic sensor networks." In Advanced Information Networking and Applications Workshops (WAINA), 2016 30th International Conference on, pp. 551556. IEEE, 2016.

[13] Majid, I. Azam, T. Khan, Z. Ali Khan, U. Qasim and N. Javaid, "A reliable and interference-aware routing protocol for underwater wireless sensor networks." In Complex, Intelligent, and Software Intensive Systems (CISIS), 2016 10th International Conference on, pp. 246-255. IEEE, 2016.

[14] Ahsan, S. Ahmed, F. Hadi, F. Wahab and I. Ahmed, "A Recent Study on Routing Protocols in UWSNs."

[15] R. Islam and Y. S. Han, "Cooperative MIMO communication at wireless sensor network: An error correcting code approach." Sensors 11, no. 10 (2011): 9887-9903.

[16] Zhou, S. Zhou, J.H Cui and S. Cui, "Energy-efficient cooperative communication based on power control and selective single-relay in wireless sensor networks." IEEE transactions on wireless communications 7, no. 8 (2008).

[17] $\mathrm{Wu}, \mathrm{W}$. Liu and $\mathrm{K}$. Li, "Power allocation and relay selection for energy efficient cooperation in wireless sensor networks with energy harvesting." EURASIP Journal on Wireless Communications and Networking 2017, no. 1 (2017): 26.

[18] Chen, M. Ma, X. Liu, A. Liu and M. Zhao, "Reliability Improved Cooperative Communication over Wireless Sensor Networks." Symmetry 9, no. 10 (2017): 209.

[19] Bravo, E. Palomar, A. Gardel and J. L. Lázaro, "Trusted and Secure Wireless Sensor Network Designs and Deployments." (2017): 1787.

[20] Gu, H. Chen, Y. Li and B. Vucetic, "Distributed multi-relay selection in accumulate-then-forward energy harvesting relay networks." arXiv preprint arXiv:1602.00339 (2016).

[21] Umar, M. Akbar, S. Ahmed, N. Javaid, Z. A. Khan and U. Qasim, "Underwater wireless sensor network's performance enhancement with cooperative routing and sink mobility." In Broadband and Wireless Computing, Communication and Applications (BWCCA), 2014 Ninth International Conference on, pp. 26-33. IEEE, 2014.

[22] Nasir, N. Javaid, H. Ashraf, S. Manzoor, Z. A. Khan, U. Qasim and M. Sher, "CoDBR: cooperative depth based routing for underwater wireless sensor networks." In Broadband and Wireless Computing, Communication and Applications (BWCCA), 2014 Ninth International Conference on, pp. 52-57. IEEE, 2014.

[23] Nasir, N. Javaid, M. Murtaza, S. Manzoor, Z. A. Khan, U. Qasim and M. Sher, "ACE: Adaptive cooperation in EEDBR for underwater wireless sensor networks." In Broadband and Wireless Computing, Communication and Applications (BWCCA), 2014 Ninth International Conference on, pp. 8-14. IEEE, 2014.

[24] Ahmed, M. Akbar, R. Ullah, S. Ahmed, M. Raza, Z. A. Khan, U. Qasim and N. Javaid, "ARCUN: Analytical approach towards reliability with cooperation for underwater WSNs." Procedia Computer Science 52 (2015): 576-583.

[25] Hafeez, N. Javaid, U. Shakeel, S. Hussain and H. Maqsood. "An Energy Efficient Adaptive Cooperative Routing Protocol for Underwater WSNs." In Broadband and Wireless Computing, Communication and Applications (BWCCA), 2015 10th International Conference on, pp. 304-310. IEEE, 2015.

[26] Javaid, H. Maqsood, A. Wadood, I. A. Niaz, A. Almogren, A. Alamri and M. Ilahi, "A localization based cooperative routing protocol for underwater wireless sensor networks." Mobile Information Systems 2017 (2017).

[27] S. Ahmed, N. Javaid, F. A. Khan, M. Y. Durrani, A. Ali, A. Shaukat, M. M. Sandhu, Z. A. Khan and U. Qasim. "Co-UWSN: cooperative energy efficient protocol for underwater WSNs." International Journal of Distributed Sensor Networks (2015). 